\title{
Analysis of some meteorological parameters using artificial neural network method for Makurdi, Nigeria
}

\author{
Chukwu, S. C. ${ }^{1 \star}$, and Nwachukwu, A. N. ${ }^{2}$ \\ ${ }^{1}$ Renaissance University, Ugbawka, Enugu, Nigeria. \\ ${ }^{2}$ University of Manchester, Manchester, United Kingdom. \\ Accepted 21 February, 2012
}

\begin{abstract}
The mean daily data for sunshine hours, maximum temperature, cloud cover and relative humidity data, were used to estimate monthly average global solar irradiation on a horizontal surface for Makurdi, Nigeria. The study used artificial neural networks (ANN) for the estimation. Results showed good agreement between the predicted and measured values of global solar irradiation. A correlation coefficient of 0.9982 was obtained with a maximum percentage error (MPE) of 0.8512 and root mean square error (RMSE) of $\mathbf{0 . 0 0 3 2}$. The comparison between the ANN and some existing empirical models showed the advantage of the ANN prediction model.
\end{abstract}

Key words: Sunshine hours, relative humidity, maximum temperature, cloudiness index, global solar radiation.

\section{INTRODUCTION}

One of the major challenges that successive regimes have faced in Nigeria is that of adequate electric power generation for her teeming population. To demonstrate the present regime of Dr. Goodluck Jonathan's determination to confront and resolve this recurring challenge head long; the Government launched a policy document called "Roadmap for Power Sector Reform", on the $26^{\text {th }}$ of August, 2010 (Federal Government of Nigeria, 2010). The document contains detailed plans of the government for solving the problem of power generation in the country. The government is to spend at least US\$3.5billion per annum for the next 10 years for power generating capacity alone. Incidentally, all the above projected financial investments in the power sector will be in the conventional non-renewable power generation sources with little or no investment in the renewable energy sources. In essence, all of Nigeria's Power Plants are fossil-fuel-fired with their attendant environmental pollution (Environment Canada, 2007).

In view of the associated environmental problems, we are of the view that the Federal Government should not neglect the renewable energy sources completely, rather researches should be encouraged in that sector as well.

\footnotetext{
*Corresponding author. E-mail: sunnychukwu007@gmail.com.
}

One of the most viable renewable energy options, particularly in Nigeria, is the abundant solar energy falling on the Earth surface. Using solar energy necessitates an exact estimation of solar energy in the proposed locations. This is usually possible through solar measurement equipments, while these devices most times are not available in some of the remote or rural locations that specially have potential for solar installation. Even in locations with these devices, the maintenance and logistics are enormous. Using prediction tools such as solar models are one of the best methods to have a good estimation of solar potential (Srivasta et al., 1993). We can categorize the solar prediction models in three distinct groups:

The first group in this classification are the empirical models. These models usually consist of few measurable meteorological parameters. Empirical models to estimate global solar radiation require the development of a set of equations that relate it to other meteorological parameters (Donatelli et al., 2003). The second categories of solar prediction models are radiative transfer models which necessitates complex geographical and meteorological parameters. Artificial neural network (ANN) models are the third and latest type of solar prediction models. These models have greater superiorities over the two prior models in terms of simplicity and accuracy (AlAlawi and Al-Hinai, 1998; Mohandes et al., 1998). 


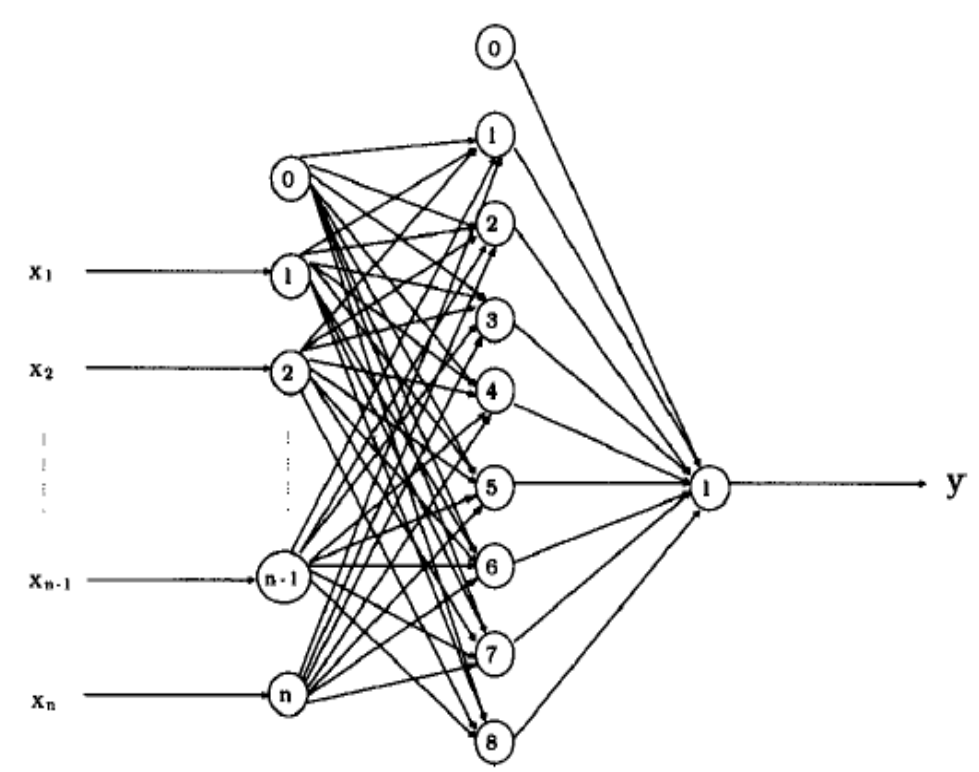

Figure 1. Structure of the Multilayered Perceptron network with single hidden layer. Node 0 is bias.

In ANN models, we can provide a prediction with a believable amount of error which is obtained with regard to more available input parameters. Also, using artificial neural network has proved its efficiency as an estimation tool for predicting factors through other input parameters which do not have any specified relationship. So applying artificial neural networks can be valuable in determining the effects of meteorological parameters and finally prediction of solar radiation. There have been several articles that have used artificial neural networks for predicting solar radiation. ANN models employ artificial intelligence techniques and are data-driven. Essentially, ANN are used to learn the behaviour of a system and subsequently used to simulate and predict this behaviour (Kalogirou, 2001). Apart from modeling solar radiation, ANN have been used in a broad range of applications including: pattern recognition and classification (Knutti et al., 2003), function approximation and prediction (Mohandes et al., 1998), identification and control (Slavisa et al., 2001), optimization and diagnostics (Reddy and Ranjan, 2003). Kalogirou (2004) has optimized a solar energy system with the purpose of maximizing the economic benefits of this system.

In this paper, Artificial Neural Network (ANN) will be used to analyze the monthly global solar radiation in Makurdi which lies in the tropics between latitudes $7.75^{\circ} \mathrm{N}$ and longitudes $8.53^{\circ} \mathrm{E}$ and altitude of $106.0 \mathrm{~m}$ above the sea level, in Benue State of Nigeria.

\section{Artificial neural network}

Artificial neural network (ANN) can be viewed as a mathematical model or computational model that is inspired by the structure or functional aspects of biological neural networks. They are characterized in principle by a network topology, a connection pattern, neural activation properties, training strategy and ability to process data. The most common neural network model is the Multilayer Perceptron (Regression, 2007; Sharda and Patil, 1990). This type of neural network is known as a supervised network because it requires a desired output in order to learn. The goal of this type of network is to create a model that correctly maps the input to the output using historical data, so that the model can then be used to produce the output when the desired output is unknown.

Figure 1 shows the block diagram of a single hidden layer multiplayer perceptron (MLP). The inputs are fed into the input layer and get multiplied by interconnection weights as they are passed from the input layer to the hidden layer. Within the hidden layer, they get summed then processed by a nonlinear function (usually the hyperbolic tangent). If more than a single hidden layer exists then, as the processed data leaves the first hidden layer, again it gets multiplied by interconnection weights, then summed and processed by the second hidden layer and so on.

Finally the data is multiplied by interconnection weights then processed one last time within the output layer to produce the neural network output. To perform any task, a set of experiments of an input output mapping is needed to train the neural network. These data are one of the most important factors to obtain reliable results from any trained ANN. Thus, the training sample data have to be fairly large to contain all the required information and must include a wide variety of data from different experimental conditions and process parameters. 


\section{MATERIALS AND METHODS}

The mean daily data for sunshine hours, maximum temperature, cloud cover, relative humidity and the global solar radiation data for Makurdi, were collected from the Archives of the Nigerian Meteorological Agency (NIMET), Federal Ministry of Aviation, Oshodi Lagos. The data obtained covered a period of twenty years (1987-2007). To develop the models, the global solar radiation data measured in millimeters using Gun-bellani distillate were converted to useful form $\left(\mathrm{MJ} / \mathrm{m}^{2} /\right.$ day) using a conversion factor of 1.1364 proposed by Sambo (1985). The first correlation proposed for estimating the monthly mean daily global solar radiation on a horizontal surface $\mathrm{H}$ (MJ m $\mathrm{m}^{-2} \mathrm{day}^{-1}$ ) using the sunshine duration data is due to Angstrom and Roy (1924). Prescott (1940) put the Angstrom correlation in more convenient form as:

$\frac{\overline{\mathrm{H}}_{\mathrm{M}}}{\overline{\mathrm{H}}_{\mathrm{O}}}=a+b \frac{\bar{n}}{\overline{\mathrm{N}}}$

Where $a$ and $b$ are regression constants, $\frac{\bar{H}_{M}}{\bar{H}_{O}}$ is the clearness index, $\bar{H}_{M}$ is the measured monthly mean daily global solar radiation, $\bar{n}$ is the monthly mean daily bright sunshine hours, $\overline{\mathrm{N}}$ is the maximum possible monthly mean daily sunshine hours or the day length, $\frac{\bar{n}}{\overline{\mathrm{N}}}$ is the fraction of sunshine hours and $\overline{\mathrm{H}}_{\mathrm{O}}$ is the monthly mean extraterrestrial solar radiation on horizontal surface given by lqbal (1983) as follows:

$\overline{\mathrm{H}}_{\mathrm{O}}=\frac{24}{\pi} \mathrm{I}_{s c} E_{\mathrm{O}}\left(\frac{\pi}{180} w_{s} \sin \phi \sin \delta+\cos \phi \cos \delta \sin w_{s}\right)$

Where, $\mathrm{I}_{s c}$ is the solar constant, $E_{\mathrm{O}}$ is the eccentricity correction factor, $\phi$ is the latitude, $\delta$ is the solar declination and $\omega_{s}$ is the hour angle. The expressions for $\mathrm{I}_{s c}, E_{\mathrm{O}}, \delta, \omega_{s}$ are given by the formulae (Liou, 1980; Robinson, 1966):

$$
\begin{aligned}
& \mathrm{I}_{s c}=\frac{1367 X 3600}{1000000}\left(\mathrm{MJm}^{-2} \mathrm{~h}^{-1}\right) \\
& \mathrm{E}_{\mathrm{O}}=1+0.033 \cos \left(\frac{360 \mathrm{~N}}{365}\right)
\end{aligned}
$$

Where, $\mathrm{N}$ is the characteristic day number for each month.

$$
\begin{aligned}
& \delta=23.45 \operatorname{Sin}\left[\frac{360 N+284}{365}\right] \\
& \omega_{s}=\operatorname{Cos}^{-1}-\tan \phi \tan \delta
\end{aligned}
$$

The average day length for each month was collected using the expression by lqbal (1983).

$$
\overline{\mathrm{N}}=\frac{2}{15} \operatorname{Cos}^{-1}-\tan \phi \tan \delta
$$

The various meteorological data are related to the global solar radiation. Table 1 shows the measured meteorological parameters.

\section{Data analysis}

i) For our analysis we used the ANN algorithm built into SPSS17 software (2008). The algorithm implements automatically the multi layer perceptron neural network with gradient descent learning.

ii) In addition, the software displays the following useful information in the output:

Network Structure - Displays summary information about the neural network.

Description - Displays information about the neural network, including the dependent variables, number of input and output units, number of hidden layers and units, and activation functions. Diagram - Displays the network diagram as a non-editable chart. We note that as the number of covariates and factor levels increases, the diagram becomes more difficult to interpret. Figure 2. Synaptic weights - Displays the coefficient estimates that show the relationship between the units in a given layer to the units in the following layer. The synaptic weights are based on the training sample, even if the active dataset is partitioned into training, testing and holdout data. Note that the number of synaptic weights can become rather large and that these weights are generally not used for interpreting network results.

Network performance - Displays results used to determine whether the model is "good". We note that charts in this group are based on the combined training and testing samples or only on the training sample if there is no testing sample.

Model summary - Displays a summary of the neural network results by partition and overall, including the error, the relative error or percentage of incorrect predictions, the stopping rule used to stop training, and the training time. The error is the sum-of-squares error when the identity, sigmoid, or h(p)erbolic tangent activation function is applied to the output layer. It is the cross-entropy error when the softmax activation function is applied to the output layer. Relative errors or percentages of incorrect predictions are displayed depending on the dependent variable measurement levels.

If any dependent variable has scale measurement level, then the average overall relative error (relative to the mean model) is displayed. If all dependent variables are categorical, then the average percentage of incorrect predictions is displayed. Relative errors or percentages of incorrect predictions are also displayed for individual dependent variables. Table 3 shows the model summary.

(iii) Depending on preferences, numerous other network information can also be displayed in Table 4.

(iv) Multiple regression analysis was employed for the empirical models (Angstrom and Roy, 1924; Prescott, 1940; Page, 1964; Swartmann and Ogunlade, 1967; Bamiro, 1983; Burari and Sambo, 2003; Augustine and Nnabuchi, 2010). The utility of the models were obtained using:

$$
R^{2}=\sum\left(\frac{H_{i, \text { meas }}-H_{i, c a l}}{H_{i, \text { meas }}-\overline{H_{i, c a l}}}\right)^{2}
$$

$R \mathrm{MSE}=\left[\sum \overline{\mathrm{H}}_{i, c a l}-\overline{\mathrm{H}}_{i, \text { meas }}{ }^{2} / n\right]^{\frac{1}{2}}$

$\mathrm{MPE}=\left[\sum\left(\frac{\overline{\mathrm{H}}_{i, \text { meas }}-\overline{\mathrm{H}}_{i, \text { cal }}}{\overline{\mathrm{H}}_{i, \text { meas }}} \mathrm{X} 100\right)\right] / n$ 
Table 1. Meteorological data and global solar radiation for Makurdi.

\begin{tabular}{|c|c|c|c|c|c|c|c|}
\hline Month & $\begin{array}{l}\overline{\mathbf{n}} \\
\overline{\overline{\mathbf{N}}}\end{array}$ & $\overline{\mathbf{T}}_{\mathrm{M}}$ & $\overline{\bar{c}}$ & $\frac{\overline{\mathbf{R}}}{\mathbf{1 0 0}}$ & $\begin{array}{c}\overline{\mathbf{H}}_{\mathrm{M}} \\
\left(\mathrm{MJ} / \mathrm{m}^{2 /} \text { day }^{1)}\right.\end{array}$ & $\begin{array}{c}\overline{\mathbf{H}}_{0} \\
\left(\mathrm{MJ} / \mathrm{m}^{2 /} \text { day }^{1}\right)\end{array}$ & $\overline{\mathbf{K}}_{\mathrm{T}}=\frac{\overline{\mathbf{H}}_{\mathbf{M}}}{\overline{\mathbf{H}}_{\mathbf{0}}}$ \\
\hline Jan & 0.560 & 37.12 & 0.0729 & 0.3660 & 16.738 & 33.03 & 0.507 \\
\hline Feb & 0.601 & 39.09 & 0.07335 & 0.3770 & 18.507 & 35.48 & 0.522 \\
\hline Mar & 0.565 & 39.35 & 0.07315 & 0.4893 & 17.884 & 37.19 & 0.481 \\
\hline Apr & 0.590 & 37.67 & 0.07265 & 0.5970 & 17.111 & 37.68 & 0.454 \\
\hline May & 0.568 & 34.93 & 0.07275 & 0.6990 & 17.192 & 37.11 & 0.463 \\
\hline Jun & 0.488 & 32.95 & 0.07295 & 0.7585 & 15.975 & 36.33 & 0.440 \\
\hline Jul & 0.377 & 31.93 & 0.07335 & 0.7805 & 13.856 & 36.54 & 0.379 \\
\hline Aug & 0.373 & 30.03 & 0.0736 & 0.7973 & 13.085 & 37.3 & 0.350 \\
\hline Sept & 0.450 & 30.77 & 0.0734 & 0.7660 & 15.203 & 37.01 & 0.412 \\
\hline Oct & 0.570 & 31.82 & 0.07265 & 0.7143 & 16.908 & 35.74 & 0.473 \\
\hline Nov & 0.706 & 33.65 & 0.0724 & 0.5843 & 18.556 & 33.04 & 0.562 \\
\hline Dec & 0.671 & 34.55 & 0.0727 & 0.4330 & 17.687 & 32.09 & 0.551 \\
\hline
\end{tabular}

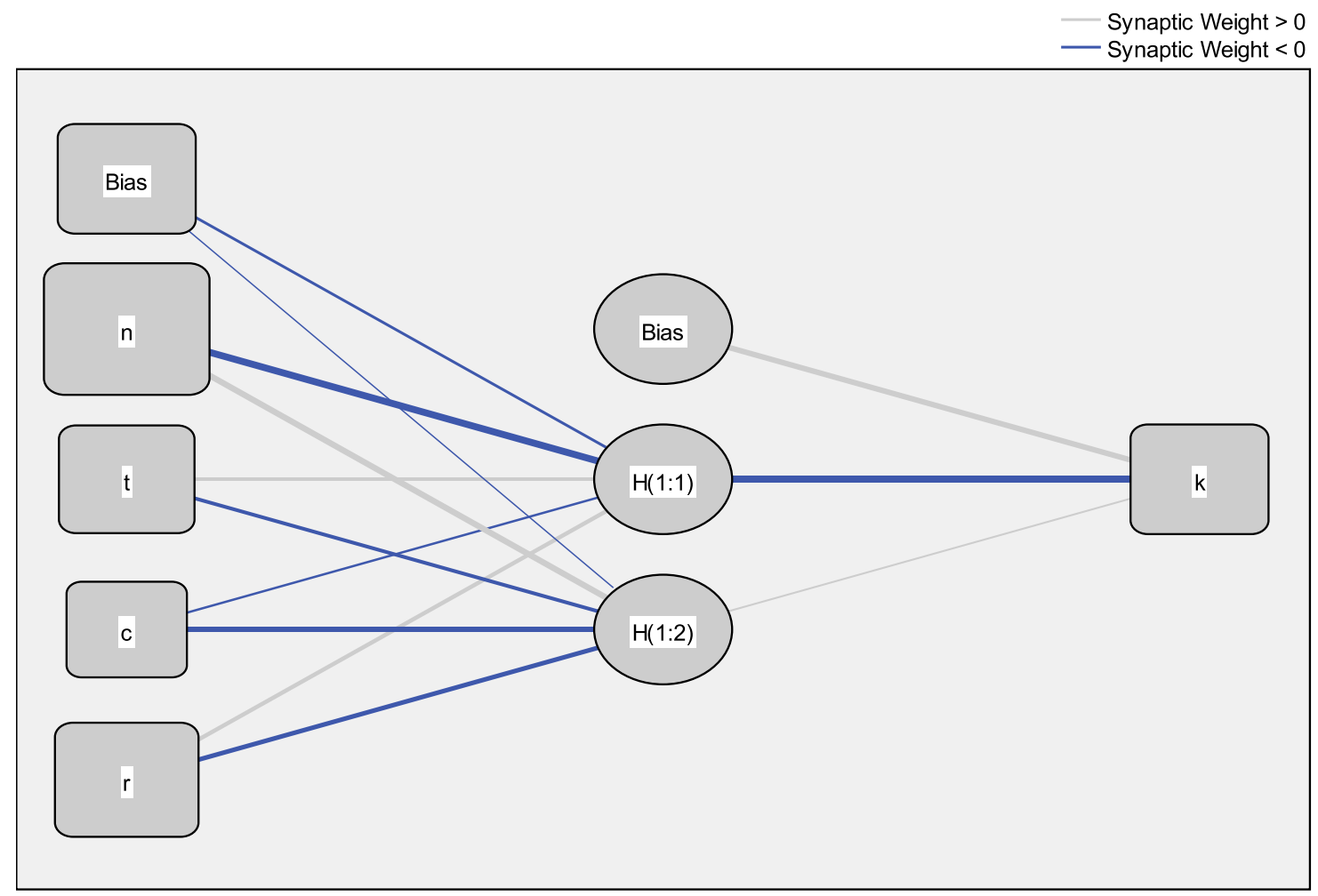

Hidden layer activation function: Hyperbolic tangent

Output layer activation function: Identity

Figure 2. Network diagram. n-represents the sunshine hour; t-represents maximum temperature; c-represents cloud cover; r-represents relative humidity and the output k-represents clearness index.

Where $\overline{\mathrm{H}}_{i, c a l}$ and $\overline{\mathrm{H}}_{i, \text { meas }}$ are the ith calculated (predicted), $\overline{H_{i, c a l}}$ is the mean of the calculated values respectively and $\mathrm{n}$ is the total number of observations.

\section{RESULTS AND DISCUSSION}

\section{Computer results for the MLP network model}

The values of the goodness-of-fit indices are shown in 
Table 2. Values for the goodness - of - fit index.

\begin{tabular}{lccc}
\hline Model & $\mathbf{R}^{2}$ & RMSE & MPE \\
\hline Angstrom-Prescott-Page & 0.2600 & 0.0820 & 22.3759 \\
Bamiro & 0.3500 & 0.0768 & 21.2233 \\
Swartmann-Ogunlade & 0.6766 & 0.0543 & 16.3304 \\
Burari and Sambo (2003) & 0.7710 & 0.0456 & 12.8941 \\
Augustine and Nnabuchi & 0.8410 & 1.5640 & 0.0770 \\
Artificial Neural Network & 0.9982 & 0.0032 & 0.8212 \\
\hline
\end{tabular}

Where $\mathrm{R}^{2}$ is the coefficient of determination, RMSE is the root-mean-square error and MPE is the mean percentage error.

Table 3. Dependent Variable: $\mathrm{k}$

\begin{tabular}{llc}
\hline & & \\
\hline & Sum of Squares Error & Model summary \\
Training & Relative Error & 0.051 \\
& Stopping Rule Used & 0.015 \\
& Training Time & 1 consecutive step(s) with no decrease in error \\
& & $0: 00: 00.000$ \\
Testing & Sum of Squares Error & \\
& Relative Error & 0.048 \\
\hline
\end{tabular}

a. Error computations are based on the testing sample.

Table 4. Hidden layer parameters

\begin{tabular}{|c|c|c|c|c|}
\hline \multicolumn{5}{|c|}{ Parameter estimates } \\
\hline & & & Predic & \\
\hline \multirow[t]{2}{*}{ Predictor } & & \multicolumn{2}{|c|}{ Hidden layer 1} & \multirow{2}{*}{$\begin{array}{c}\text { Output layer } \\
\mathbf{k}\end{array}$} \\
\hline & & $H(1: 1)$ & $H(1: 2)$ & \\
\hline \multirow{5}{*}{ Input layer } & (Bias) & 1.044 & -0.511 & \\
\hline & $\mathrm{n}$ & 1.021 & 1.437 & \\
\hline & $\mathrm{t}$ & 0.338 & -0.537 & \\
\hline & c & 0.018 & 0.293 & \\
\hline & r & 0.243 & -0.508 & \\
\hline \multirow{3}{*}{ Hidden layer 1} & (Bias) & & & -0.170 \\
\hline & $H(1: 1)$ & & & 0.747 \\
\hline & $\mathrm{H}(1: 2)$ & & & 0.847 \\
\hline
\end{tabular}

\section{RESULTS AND DISCUSSION}

\section{Computer results for the MLP network model}

The values of the goodness-of-fit indices are shown in Table 2. The values of the coefficient of determination show poor correlation between the measured and the predicted values for Angstrom-Prescott-Page and Bamiro models. These models could not be applied for prediction of global solar radiation in Makurdi. Fair correlation was obtained using Swartmann and Ogunlade (1967), Burari and Sambo (2003) and Augustine and Nnabuchi (2010) models. The Burari and Sambo (2003) model and Augustine and Nnabuchi model could be used for shortterm prediction of solar radiation in Makurdi. The Burari and Sambo model gave the value of root-mean-square error of 0.0456 . This model could not be used for longterm prediction of global solar radiation in Makurdi. The 


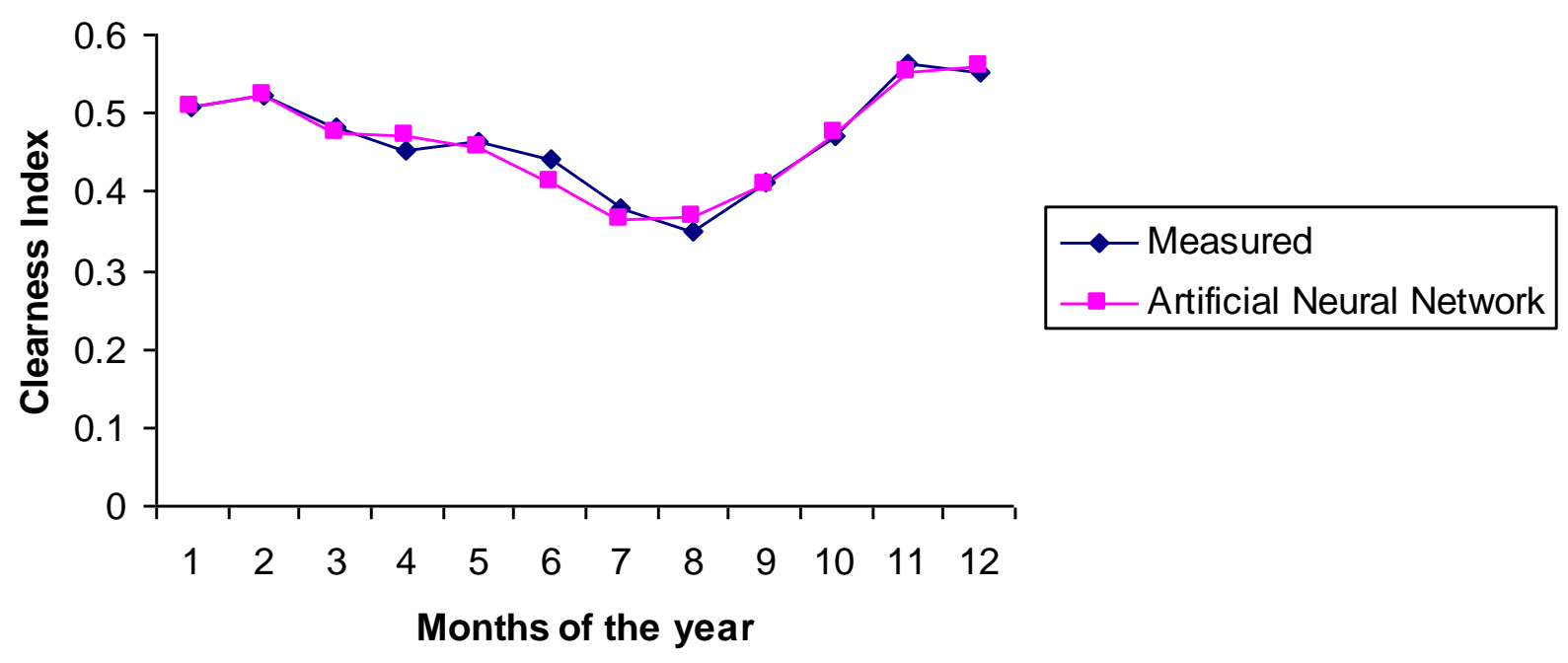

Figure 3. A graph of measured and predicted values of clearness index for Makurdi.

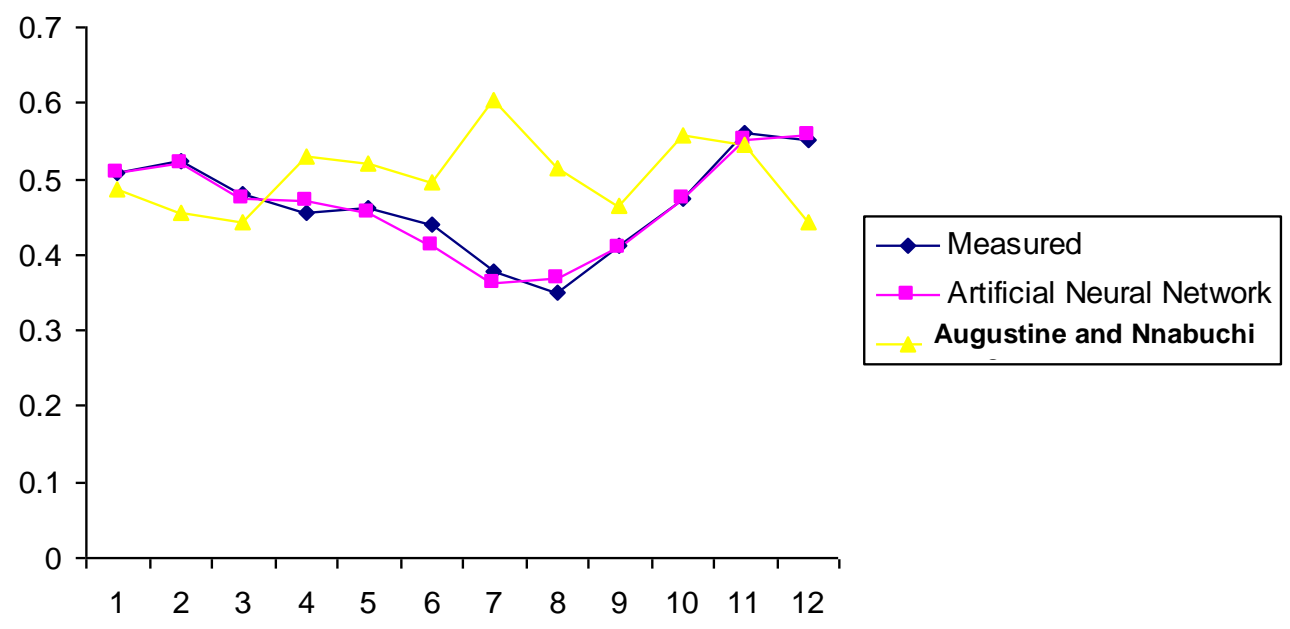

Figure 4. Comparison measured, artificial neural network and empirical models.

Augustine and Nnabuchi model gave a good correlation with values of the coefficient of determination of 0.8410 , root-mean-square error of 1.564 and maximum percentage error of 0.077 . The graphs for the measured and predicted values of the clearness index for the various models are shown in Figures 3 and 4 . In the figures, each model is compared with the ANN model.

The best empirical model is the Augustine and Nnabuchi model as can be seen in Table 2 and Figures 3 and 4. When ANN is applied to the data from Makurdi an improved coefficient of determination, root-mean-square error and maximum percentage error were obtained as shown in Table 2. The graph of predicted and measured values of the clearness index is shown in Figures 3 and 4. This study has confirmed the results obtained by AlAlawi and Al-Hinai (1998), Mohandes et al. (1998), Reddy and Ranjan (2003) and AbdulAzeez (2011).

\section{Conclusion}

Four meteorological parameters were used for the analysis of solar irradiation in Makurdi, Benue State of Nigeria. Artificial Neural Network was employed to obtain the predicted values of the average monthly solar radiation. The result shows an excellent agreement between measured and predicted values with coefficient of determination of 0.9982 , maximum percentage error of 0.8212 and root-mean-square error of 0.0032 . The comparison between the ANN model and some existing empirical models has shown the superiority of the ANN model. The result has confirmed the results from similar application of the ANN model in the prediction of solar radiation.

The field of neural networks is very diverse and opportunities for future research exist in many aspects, 
including data pre-processing and representation, architecture selection, and application (Bishop, 1995).

The next logical step for the research is to improve further the performance of Neural Networks, for this application, perhaps through better training methods, better architecture selection, or better input.

\section{REFERENCES}

Abdulazeez MA (2011). Artificial neural network estimation of global solar radiation using meteorological parameters in Gusau, Nigeria. Archiv. Appl. Sci. Res., 3(2): 586-595.

Al-Alawi SM, Al-Hinai HA (1998). An ANN based approach for predicting global solar radiation in locations with no direct instrumentation. Renewable Energy, 14: 199-204.

Angstrom AJ, Roy QJ (1924). Solar and Terrestrial Radiation. Met. Soc., 50:121-126.

Bamiro OA (1983). Empirical relations for the determination of solar radiation in Ibadan, Nigeria. Solar Energy, 31: 85-94.

Bishop CM (1995). "Neural Networks for Pattern Recognition," Oxford University Press.

Burari FW, Sambo AS (2003). Analysis of monthly average daily global radiation for Bauchi. J. Trop. Eng., 4(1): 26-31.

Donatelli M, Bellocchi G, Fontana F (2003). Software to estimate daily radiation data from commonly available meteorological variables. Agric. For. Meteorol., 18:363-367.

Environment Canada (2007). 'Impacts of Power Generation on the Environment'. Internet News .(www.weatheroffice.gc.ca).

Igbal M (1983). An introduction to solar radiation. Academy Press. New York.

Federal Government of Nigeria (2010). Roadmap to Power Sector Reform. A customer- driven sector- wide plan to achieve stable power supply. A policy document of the Federal Government of Nigeria.
Kalogirou SA (2001). Artificial neural networks in renewable energy systems application: a review. Renewable Sustainable Energy Rev., 5: $373-401$

Kalogirou SA (2004). Optimization of solar systems using artificial neural network and genetic algorithms. Appl. Energy, 77: 383-405.

Knutti R, Stocker TF, Joos F, Plattner GK (2003). Probabilistic climate change predictions using neural networks. Climate Dynamics, 21: 257-272.

Liou KN (1980). Introduction to Atmospheric Radiation. Academy Press, New York.

Mohandes M, Rehman S, Halawani TO (1998). Estimation of global solar radiation using artificial neural network. Renewable Energy. 14:179-84.

Page JK (1964). Environmental research using models .Proc. UN. Conf. New sources of Energy. 4: 378.

Prescott JA (1940). Evaporation from a water surface in relation to solar radiation, Tran. R. Soc. S. Austr., 64: $114-118$.

Reddy KS, Ranjan M (2003). Solar resource estimation using ANN and comparision with other correlation models. Energy Conversion Manage., 44: 2519-2530.

Robinson N (1966). Solar Radiation. Elsevier Publishing Company, New York.

Sharda R, Patil R (1990). "Neural Networks as forecasting experts: an empirical test," Proceedings of the 1990 Int. Joint Conf. Neural Networks, I: 491-494, Washington DC, USA.

Srivasta SK, Singh OP, Pandey GN (1993). Estimation of global solar radiation in uttah Pradesh (India) and comparison of some existing correlations. Solar Energy. 51:27-29.

Swartman RK, Ogunlade O (1967). Solar radiation estimatesfrom common parameters. Solar Energy, 11: 170 - 172.

SPSS17 software (2008). Spss predictive analytics software and solutions. SPSS Inc., http://www.spss.com. 